\title{
MULTI-BANDS IMAGE SEGMENTATION: A SCALAR APPROACH
}

\author{
Chafik KERMAD, Kacem CHEHDI
}

\author{
LASTI - ENSSAT, University of Rennes I \\ 6 rue de Kerampont, BP 447, 22305 Lannion Cedex, France \\ e-mail: kermad@enssat.fr, chehdi@enssat.fr
}

\begin{abstract}
In the domain of multi-bands image processing, two different approaches can be considered: the scalar one and the vectorial one. This paper presents a method that belongs to the first approach. The method is achieved in three steps. The first step tempts to eliminate redundant observations by making a selection of relevant bands. In the second step, each of the selected bands is segmented using a technique of histogram multi-thresholding. In the last step, a fusion by a combination of the results of the selected bands allows to obtain the final segmentation. This scheme is illustrated in the frame of an application in high-resolution multispectral imagery acquired by the Compact Airborne Spectrographic Imager (CASI).
\end{abstract}

\section{INTRODUCTION}

In order to collect a maximum of information from a scene, the images currently used are generally of multi-bands nature. The segmentation of these images is an important research theme that finds many applications in color, multispectral, or multi-temporal imagery [1] [3] [4] [5] [7] [8] [10] [11] [12]. Two different schemes can be envisaged to solve this problem: $(i)$ The scalar processing scheme, that consists in processing each band separately and then in proceeding to a fusion step of the results. (ii) The vectorial processing scheme, that considers each pixel as a vector and consists in applying a single processing taking explicitly account of the multidimensional nature of data.

In this paper we present, in a framework of airborne multispectral imagery, an example of practice utilization of the scalar segmentation approach. This alternative to the vectorial approach appears well adapted to the examined problem.

\section{DEVELOPED METHOD}

The principle of the proposed method consists in processing separately the relevant bands of the multispectral image, and then in proceeding to a fusion of the results obtained on these bands. Informations contained in these images are often complementary but present sometimes important redundancies. The first step of processing consists in making a selection of relevant bands. Each of the spectral bands is first characterized by a global histogram built from significant peaks of local histograms. An aggregation by using the global histograms allows then to form a set of classes of similar bands by maximizing an entropy criterion. The representative band of each class is that minimizing a dissimilarity measure with the center of the class. Each of the selected bands is then segmented by using a technique of histogram multi-thresholding. This one is achieved by an iterative gray levels aggregation operating on the global histogram of the band. At last, a fusion that combines the multi-thresholding results of the selected bands allows to obtain the final segmentation.

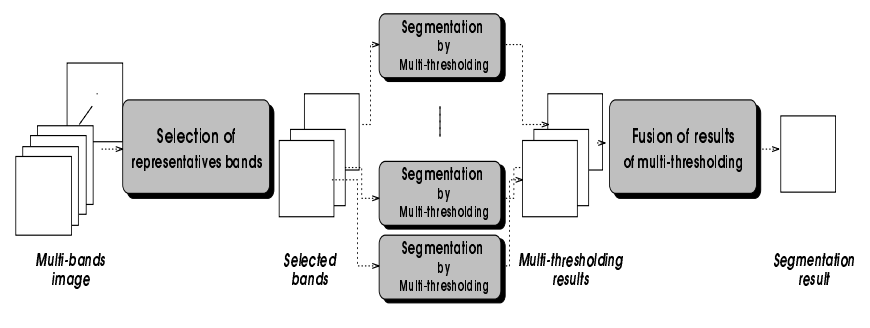

Fig. 1. The segmentation approach synopsis.

We present in what follows these three steps as well as experimental results obtained in the framework of an application concerning the detection and the classification of seaweed deposit zones by aerial multispectral image analysis.

\subsection{Relevant bands selection}

In multi or hyperspectral data, neighboring bands frequently appear similar and convey often same information. The interband correlation is due to the spectral proximity. An analysis of the multi-bands images is then necessary to reduce these redundancies. One method to realize this is through principal component analysis. This technique is interesting but poses certain problems when the correlation matrix of 
the spectral bands is ill-conditioned. The accumulation of errors in the calculation of this one can be considerable, what renders the precise estimation of its eigenvalues and eigenvectors delicate. Moreover, if the size and the number of spectral bands increase, the dimension of the matrix increases equally and the computational cost becomes important. In this paper, we propose a different approach in which the relevant band choice is made among that existent. This selection is realized in two phases. Each of the bands is first characterized by a global histogram. Similar bands aggregations followed by a selection of the representative band of each of the formed classes are then undertaken.

\subsubsection{Construction of the global histogram}

This first phase leans on a transformation of the local histograms and on simple decision criteria to determine local significant peaks that will serve for the construction of the global histogram.

\section{Transformation of the local histograms:}

The procedure of transformation is necessary because most multi-modal local histograms have modes with badly defined limits. The calculation of the maxima is then delicate. To put in obviousness the principal modes of each histogram, we use four criteria based on the measure of gray level cooccurences and on a function of Sensitivity of the Eye to the Contrast (SEC) [9]. The introduction of this function allows to take into account the visibility of a point as compared to its neighbors. It favors the aggregation of points with visually close illuminations. In function of the number of verified criteria, the gray level occurrence frequencies are more or less pondered.

\section{Significant peaks detection:}

Significant peaks of the histograms resulting from the previous transformations have to verify three criteria: $(i)$ A gray level is a peak of the histogram if it presents a maximal local occurrence frequency. (ii) The distance separating two significant peaks has to be superior to a threshold $s_{d}$; otherwise we retain as significant peak that whose occurrence frequency is the greatest. (iii) The height of a peak has to be superior to a fraction $s_{l}$ of the maximum of the histogram. This allows to eliminate peaks of relatively weak occurrence frequencies. The values of the thresholds $s_{d}$ and $s_{l}$ are calculated in an automatic manner and represent the average of distances of successive peaks couples, and the average of appearance frequencies of peaks.

The occurrence frequencies of the valid peaks are drawn in a global histogram. This one, noted $H_{\text {glob }}$, presents a wellpronounced multi-modal form. This phase is realized by using several sizes of windows $(16 \times 16,32 \times 32,64 \times 64)$ so as to take into account the spatial resolution of the image. The occurrence frequencies of the peaks determined by applying the different sizes are drawn in the same global histogram.

\subsubsection{Aggregation and selection of representative bands}

The aim of this phase is to select relevant bands that will be segmented in the following step. First, an aggregation of the bands by an analysis of the global histograms allows to form classes of similar bands. Then, the representative band of each class is selected by seeking that minimizing a dissimilarity measure with the center of the class.

Aggregation of bands:

At the begining, each band is associated with a different class. At each step, the two closest classes, in the sense of a measure of dissimilarity, are regrouped. A band $B_{u}$ characterized by its global histogram $H_{u}=\left(u_{1}, u_{2}, \ldots, u_{N}\right)$ is then regrouped with an other band $B_{v}$ modeled by the global histogram $H_{v}=\left(v_{1}, v_{2}, \ldots, v_{N}\right)$ if they minimize the normalized dissimilarity measure defined by:

$$
d_{T}\left(H_{u}, H_{v}\right)=1-\frac{\sum_{i} u_{i} v_{i}}{\sum_{i} u_{i}{ }^{2}+\sum_{i} v_{i}{ }^{2}-\sum_{i} u_{i} v_{i}}
$$

The dissimilarity measure between two formed classes of several bands (inter classes dissimilarity) is equal to the average of the dissimilarities between the bands of the two classes. In practice, the determination of the number of classes to form and the thresholds on the dissimilarity measure is sometimes very delicate. In order to make the aggregation process unsupervised, we propose the utilization of a measure of entropy type that allows to evaluate the homogeneity of the set of the obtained classes after each aggregation. The retained bands grouping is that of the set which maximizes this entropy.

In information theory, the entropy associated with a set of possible events is $E=-\sum_{i} p_{i} \ln p_{i}$, where $p_{i}$ is the probability of occurrence of the event $i$. Maximizing the entropy returns to distribute a rare quantity between several sets, in a manner the most uniform possible. In our case, that is translated by the fact to form classes having a common criterion the most equitable possible. This criterion can be based on the probability that an unknown band is classified in one of the formed groups by the aggregation algorithm [6]. We therefore seek to make some groups whose the aggregation probability is the same. Several hypotheses to evaluate this probability are possible.

In the case where the bands are to be distributed in an uniform manner in the "space of bands", the probability $p_{i}$ to a new band to belong to one of the classes can therefore be proportional to the size of this class. This size can be estimated by the average dissimilarity between the bands of this class (intra class dissimilarity).

The hypothesis of uniformity is not general, a modification 
of probabilities is necessary to take into account the fact that some very close bands can provide a class as interesting as a weaker number of bands distributed in a distant area of the space. That is translated by making $p_{i}$ not only proportional to the size of the class, but also to the number of bands that compose it. To obtain probabilities, a factor of normalization is added. That, at last, gives:

$$
p_{i}=\frac{n_{i} d_{i i}}{\sum_{i} n_{i} d_{i i}+\frac{1}{n} \sum_{i \neq j} d_{i j}}
$$

where $n_{i}$ is the number of bands of the class $i, d_{i i}$ is the intra class dissimilarity, $d_{i j}$ is the inter classes dissimilarity and $n$ is the number of the obtained classes.

\section{Representative bands selection:}

Each of the previously formed classes is characterized by its mean histogram $H_{g}$. The representative band of the class is that one having the closest global histogram to $H_{g}$ in the sense of the employed dissimilarity measure.

\subsection{Scalar segmentation}

The selected bands are segmented using an histogram multithresholding technique [9]. The multi-thresholding operates on the global histogram $H_{g l o b}$ of the band. It is realized by an iterative procedure allowing to aggregate the masses of $H_{\text {glob }}$ into significant punctual masses characterizing the regions that compose the band. The aim is to detect the most significant thresholds $s_{g}$ by displacing iterativly the masses to their gravity centers until the convergence of histograms. This procedure is described hereafter.

Let $H^{(0)}(i)=H_{\text {glob }}(i), i \in\left[i_{\min }, i_{\max }\right]$, be the initial histogram to process, and $H^{(k)}$, be that one obtained at the iteration $k$. The Frequencies of occurrence of the gray levels at the iteration $k+1$ are determined according to following relation:

$$
H^{(k+1)}(i)=\sum_{s \in V_{i}} H^{(k)}(s) \delta\left(i-\mu_{k}(s)\right)
$$

where $\delta($.$) represents the symbol of Kronecker and \mu_{k}(s)$ designates the average at the iteration $k$ defined by:

$$
\mu_{k}(s)=\frac{\sum_{s \in V_{i}} s H^{(k)}(s)}{\sum_{s \in V_{i}} H^{(k)}(s)}
$$

$V_{i}$ is the set of the $2 m+1$ gray levels neighbors of $i$ :

$$
V_{i}=\left\{\max \left((i-m), i_{\min }\right) \ldots \min \left((i+m), i_{\max }\right)\right\}
$$

The relation (3) translates the displacement of a part of the masses of $H^{(k)}(i)$ to their local centers of gravity. To make the procedure automatic, we vary gradually $m$ until successive sizes provide the same results. The obtained final histogram is constituted by punctual masses. To each mass corresponds a class and the position of each mass indicates the gray level that will be assigned as label to each class. The values of thresholds $s_{g}$ are given by the barycenters of the gray levels determined by the position of these masses. They allow to define the intervals of illumination of the classes and to associate a label to each point of the band.

\subsection{Fusion of multi-thresholding results}

This step consists in combining results issued from the previously segmented bands so as to produce a representation better than those of the results taken separately. It does not exist a standard method allowing to realize this operation. Various techniques can be employed, going from usual statistical algorithms, as bayesian methods, until ad hoc means, as the vote, passing by more recent techniques based on neural networks systems or on the fuzzy set theory [2]. In our case, we have used a vectorial classification. The result of the fusion is then obtained by the aggregation of the combinations of labels of the multi-thresholded bands following an unsupervised k-means approach.

\section{EXPERIMENTAL RESULTS}

In the framework of studies undertaken by the Remote sensing Group in Brittany (GSTB) in France, we have been interested in the problem of location and classification of zones of seaweed deposits by the analysis of multispectral images in order to evaluate the seaweed covering by surface unit. The tested image was collected by the CASI sensor on July 1998, and has a 2 metre resolution. It is composed of ten spectral bands $(1024 \times 512)$ corresponding to ten different wave lengths going from the visible to the near-infrared. For this image, we have at our disposal a ground truth map gevin estimations of true rate of seaweed covering in some representative sites. This has allowed to measure the validity of the proposed approach.

Three classes of similar bands were obtained by the aggregation process. The images in figure 2(a) represent the selected bands. We can notice the clear difference and thus the complementary of the selected bands. The multi-thresholding results of these three bands are represented in the figure 2(b). In examining these results, we can state that, in the sense of the gray level, the different regions, which compose the original bands, are well detected. Their boundaries align closely with the visible transitions and the details are not lost. The final classification results obtained following the developed method (figure 3(b)) are coherent as compared to those of the ground truth. These results have proven closer to the ground truth that those obtained following a vectorial approach of $\mathrm{k}$ means applied on the original bands (figure 3(c)). This one emphasizes some remarkable fluctuations due to the inconcideration of spatial relationships between pixels. 


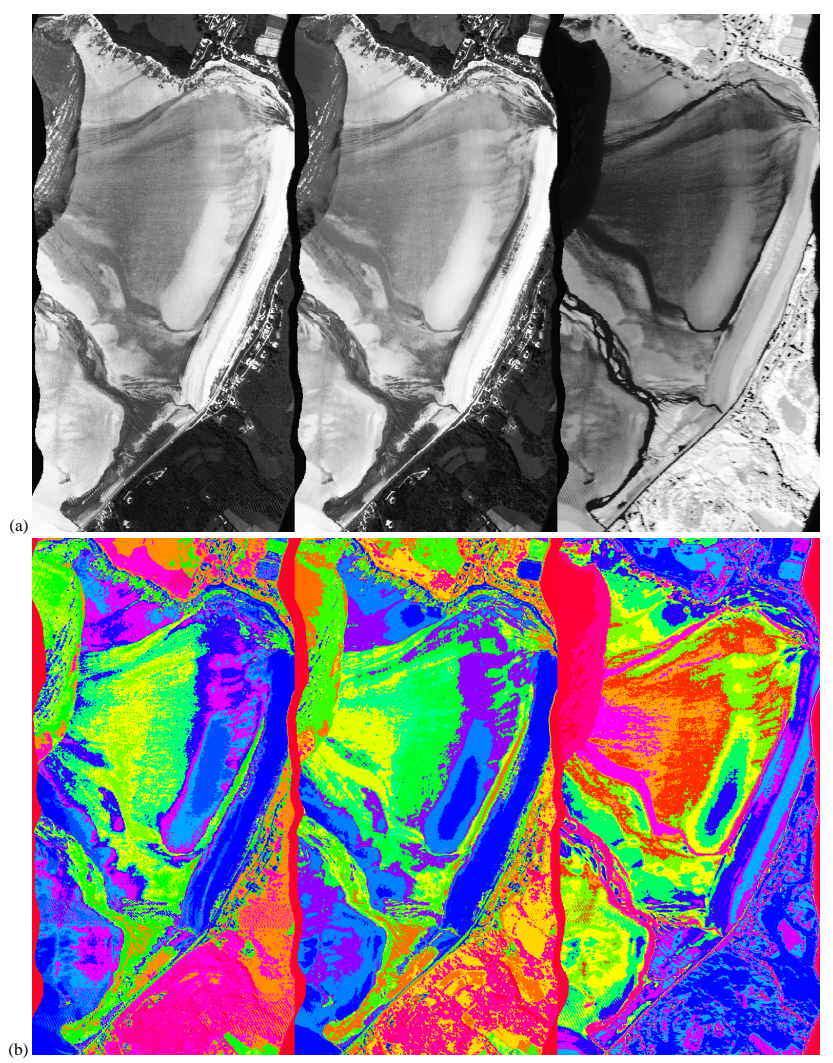

Fig. 2. (a) The selected bands. (b) The multi-thresholding results (in false colors) of the selected bands.

\section{CONCLUSION}

The suggested method allows a segmentation of multispectral images through a scalar approach. It proceeds in three steps. The first tempts to eliminate redundant observations by maximizing an entropy criterion. Scalar segmentations via an automatic multi-thresholding technique are applied on relevant bands, in the second step. Finally, a fusion of the multi-thresholding results is achieved in the last step to provide the final segmentation.

The developed approach is quasi automatic and operates without intervention of high-level knowledge. The efficiency of this approach expresses itself in the majority of examined cases, through a coherent detection of the representative elements of the images.

\section{REFERENCES}

[1] J. Bennett and A. Khotanzad, Multispectral random field models for synthesis and analysis of color images, IEEE Trans. on PAMI, vol. 20(3) 327-332, 1998.

[2] I. Bloch, Information combination operators for data fusion, IEEE Trans. on SMC, vol. 26(1) 52-67, 1996.
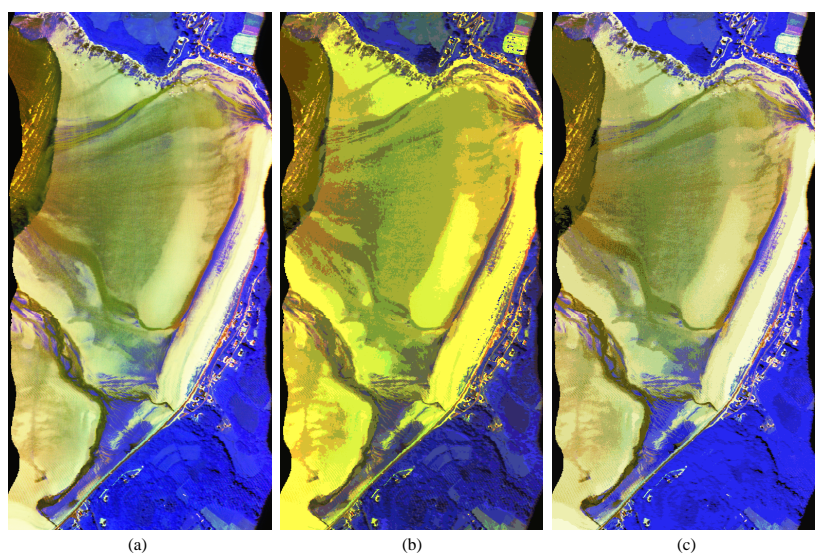

Fig. 3. (a) RGB composition of the selected original bands. (b) Result obtained by the developed approach. (c) Result obtained following a vectorial k-means classification applied on the original bands.

[3] B. Bhanu, P. Symosek, and S. Das, Analysis of terrain using multispectral images, Pattern Recognition, vol. 30(2) 197$215,1997$.

[4] J. Desachy, V. Bessettes and P. Dherete, Information processing for remote sensing, Eds, C.H. Chen, World Scientific Publishing Co. Oct. 1999.

[5] I.R. Greenshields, Coherent computation of the multispectral maximal directional derivative, Image and Vision Computing, vol. 18(1) 1-7, 1999.

[6] P. Gros, "De l'appariement à l'indexation des images". HDR thesis, INP Grenoble 1998.

[7] S. Kamata, M. Niimi, and E. Kawaguchi, Multi-temporal classification of multispectral images using a neural network, Proc. 12th ICPR vol. 2, 470-472, 1994.

[8] I.B. Kerfoot and Y. Bresler, Theoretical analysis of multispectral image segmentation criteria, IEEE Trans. on IP, vol. 8(6) 798-820, 1999.

[9] C.D. Kermad and K. Chehdi, Image segmentation: towards an automatic framework through methods cooperation, (in french) Traitement du Signal, vol. 15(4) 321-336, 1998.

[10] D. Landgrebe, Multispectral data analysis: an overview past, present, and future. Proc. of the IGARSS'95, Italy, july 1995.

[11] P.P. Raghu and B. Yegnanarayana, Multispectral image classification using gabor filters and stochastic relaxation neural network, Neural Networks, vol. 10(3) 561-572, 1997.

[12] W.E. Reddick, J.O. Glass, E.N. Cook, T.D. Elkin, and R.J. Deaton, Automated segmentation and classification of multispectral magnetic resonance images of brain, IEEE Trans. on MI, vol. 16(6) 911-918, 1997. 